T. Schmidt, B. Babic, C. J. Bruns, H. F. Fuchs

Klinik und Poliklinik für Allgemein-, Viszeral-, Tumor- und Transplantationschirurgie, Uniklinik Köln, Köln, Deutschland

\title{
Chirurgische Therapie des Ösophaguskarzinoms - neue Technologien, moderne Konzepte
}

\section{Hintergrund}

Das Ösophaguskarzinom wird in Deutschland meist in spezialisierten Zentren entsprechend den Leitlinien multimodal und interdisziplinär therapiert [1]. In Studien kommen zunehmend innovative Konzepte zur Neoadjuvanz, Adjuvanz oder zu perioperativen Therapien zum Einsatz, zusätzlich werden Konzepte ohne Chirurgie (,active surveillance“) untersucht [2, 3]. Daneben werden die etablierten Therapiekonzepte mit einer neoadjuvanten Radiochemotherapie (CROSS[Carboplatin/ Paclitaxel]-Protokoll) und einer perioperativen Chemotherapie (FLOT[5-Fluorouracil, Leucovorin, Oxaliplatin, Docetaxel]Protokoll) verglichen und es wurde die adjuvante Immunthe-

Dieser Beitrag erschien zuerst in Der Chirurg 2021 · 92:1100-1106. https://doi.org/10.1007/s00104-021-01525-3

\section{Redaktion:}

Stephan Schmitz, Köln

best practice onkologie $2022 \cdot 17$ (1-2): 42-49

https://doi.org/10.1007/s11654-022-00370-y

Online publiziert: 21. Januar 2022

(c) The Author(s), under exclusive licence to Springer Medizin Verlag $\mathrm{GmbH}$, ein Teil von Springer Nature 2022 rapie mit Nivolumab bei Patienten nach neoadjuvanten Radiochemotherapie etabliert.

Im kurativen und meist multimodalen Setting ist die thorakoabdominale Ösophagektomie mit Schlauchmagenhochzug das operative Standardverfahren [1,4]. Aufgrund der technischen Besonderheiten wird dieser zunehmend minimal-invasiv durchgeführte Eingriff als chirurgisch anspruchsvoll angesehen, und wegen der bald umzusetzenden Mindestmengenregelung von 26 Eingriffen pro Jahr wird die Zentralisierung in den nächsten Jahren nochmals zunehmen [5]. Dieser Eingriff geht auch heute noch mit einer hohen postoperativen Morbidität mit bis zu $60 \%$ einher [6]. Durch eine Zentralisierung konnte die postoperative Komplikationsrate in spezialisierten Highvolume-Zentren bereits gesenkt werden [7].

Bei der roboterassistierten Chirurgie („robotic-assisted surgery“, RAS) bedient ein Chirurg an einer Konsole ferngesteuerte Roboterarme, die die Durchführung laparoskopischer Verfahren erleichtern [8]. Der Boom der RAS hat in Nordamerika nach Zulassung des Da Vinci-Systems Anfang der 2000er-Jahre begonnen, kurze Zeit später führte Horgan im Jahre 2003 in Chicago dann die erste roboterassistierte Ösophagusresektion durch [9]. Analog zur Entwicklung in den USA setzt sich das System in Europa meist zuerst in urologischen und aktuell zu- 


\section{Chirurgische Therapie des Ösophaguskarzinoms - neue Technologien, moderne Konzepte}

Zusammenfassung

Das Ösophaguskarzinom wird in Deutschland meist in spezialisierten Zentren entsprechend den Leitlinien multimodal und interdisziplinär therapiert. In den kommenden Jahren wird die Zentralisierung der Ösophaguschirurgie in Deutschland durch die Festlegung neuer Mindestmengen weiter voranschreiten. Dieser Artikel soll neue Technologien für die chirurgische Therapie des Ösophaguskarzinoms und zudem aktuelle onkologische Konzepte aus der Sicht eines Highvolume-Centers vorstellen.

Schlüsselwörter

Mindestmengen · High-volume-Center · Minimalinvasive Chirurgie ·

Fluoreszenzlaparoskopie · Robotik

\section{Surgical treatment of esophageal cancer-New technologies, modern concepts}

\section{Abstract}

In Germany esophageal cancer is mostly treated in specialized centers according to national and international guidelines in a multimodal and interdisciplinary setting. In the next few years centralization of esophageal surgery will continue in Germany due to new national regulations on minimum case volumes. This article highlights new technologies for surgical treatment of esophageal cancer and also depicts the current oncological concepts from the perspective of a highvolume center.

\section{Keywords}

Minimum case volumes $\cdot$ High-volume center $\cdot$ Minimally invasive surgery $\cdot$ Fluorescence laparoscopy $\cdot$ Robotics nehmend in viszeralchirurgischen Abteilungen durch [10]. In jüngerer Zeit wurden oder werden andere konkurrierende Geräte auf den Markt gebracht wie z. B. das Versius-System von CMR oder das Hugo-System von Medtronic [7].

\section{》) RAS stellt neue Anforderungen an das chirurgische Training}

Die RAS hat neue Herausforderungen und zusätzliche Verantwortlichkeiten für das Training chirurgischer Teams in einem bereits anspruchsvollen und facettenreichen Arbeitsumfeld geschaffen. Dies bezieht sich auf die komplexe, hochtechnische Ausrüstung, die Patientenpositionierung, die lange Dauer des Eingriffs, die Trennung des Konsolenchirurgen vom Patienten. Mit diesen Herausforderungen hat sich unsere Arbeitsgruppe zusammen mit einem weltweiten Panel von Roboterchirurgen beschäftigt und im Rahmen eines Delphi-Prozesses konkrete Verfahrensanweisungen publiziert [11]. Neben den hochkomplexen und teuren RAS-Master-and-Slave-Systemen haben sich jüngst mehrere Anbieter von Laparoskopiesystemen mit roboterähnlichen Eigenschaften vornehmlich im Hinblick auf Triangulationsmöglichkeiten platziert [12-14].

Ziel dieser Arbeit ist die Darstellung ausgewählter neuer Technologien und Konzepte für die Behandlung des Ösophaguskarzinoms aus der Sicht eines Exzellenzzentrums für die Chirurgie des oberen Gastrointestinaltraktes.

\section{Neue onkologische Behandlungskonzepte}

Das Ösophaguskarzinom ist durch die anatomische Lage definiert und fasst mit dem Plattenepithelkarzinom und dem Adenokarzinom des Ösophagus bereits zwei komplett unterschiedliche Erkrankungen zusammen. Plattenepithelkarzinome sind Plattenepithelkarzinomen in anderen Organen ähnlicher als den Adenokarzinomen des Ösophagus [15].
In Europa werden Patienten mit lokal fortgeschrittenen Ösophaguskarzinomen mit multimodalen Therapiekonzepten behandelt. Hierbei werden insbesondere das neoadjuvante CROSS-Konzept mit einer Radiochemotherapie für sowohl Plattenepithelkarzinome und Adenokarzinome als auch das perioperative FLOT-Therapiekonzept mit einer neoadjuvanten und adjuvanten Chemotherapie bei Adenokarzinomen eingesetzt [16, 17]. Beide Therapiekonzepte werden aktuell in Deutschland in der randomisierten ESOPEC-Studie miteinander verglichen [18]. In Neo-AEGIS (Neoadjuvant Trial in Adenocarcinoma of the Esophagus and Esophgo-Gastric Junction International Study) wurde ein ähnlicher Ansatz gewählt und das frühere MAGIC-Chemotherapie-Protokoll mit dem CROSS-Protokoll verglichen. Ursprünglich sollte eine Überlegenheit des CROSS-Protokolls bewiesen werden. Allerdings wurde die Studie nach den Ergebnissen der FLOT4-Studie modifiziert und auch eine perioperative FLOT-Behandlung ab 2019 zugelassen. Die aktuellen Daten der Interimsanalysen zeigen keinen Unterschied im 3-Jahres-Überleben zwischen den beiden Gruppen, sodass das Data Safety Monitoring Board im Dezember 2020 eine Beendigung der Rekrutierung empfohlen hat [19].

\section{\) Nivolumab bindet an den PD-1-Rezeptor auf T-Zellen und stimuliert das Immunsystem}

Dieses Jahr wurde nach einer neoadjuvanten Radiochemotherapie eine adjuvante Immuntherapie mit dem Checkpoint-Inhibitor Nivolumab bei Patienten ohne komplette pathologische Response durch die US Food and Drug Administration (FDA) und die European Medicines Agency (EMA) in Europa zugelassen. Nivolumab ist ein monoklonaler Antikörper und bindet an den PD-1(„programmed cell death protein 1“)-Rezeptor auf T-Zellen und stimuliert so das Immunsystem. In der CHECKMATE-577-Studie wurde Nivolumab in einer 2:1-Ran- 
domisierung für ein Jahr mit einer Placebogabe bei Patienten mit kurativ operierten Ösophaguskarzinomen nach stattgehabter neoadjuvanter Radiochemotherapie verglichen. Bei den 532 mit Nivolumab behandelten Patienten betrug das mediane krankheitsfreie Überleben 22,4 Monate, während die 262 mit Placebo behandelten Patienten ein krankheitsfreies Überleben von 11,0 Monaten hatten. Der Überlebensvorteil war unabhängig von der Tumorentität und der PD-L1-Expression [20]. Durch diese Studie wurden die Therapieoptionen für CROSSvorbehandelte Patienten deutlich erweitert. Aktuell läuft noch eine Vielzahl weiterer Studien, bei denen eine Immuntherapie in der perioperativen Behandlung untersucht wird.

Ein weiteres Behandlungskonzept, das aktuell diskutiert wird, ist die aktive Beobachtung („active surveillance“) von $\mathrm{Pa}$ tienten mit Ösophaguskarzinom, die ein komplettes klinisches Ansprechen auf eine neoadjuvante Radiochemotherapie zeigen. Hierzu wird in den Niederlanden die SANO(Surgery as Needed of Oesophageal Cancer)-Studie durchgeführt. In dieser PhaseIII-Studie wird eine aktive Beobachtung mit einer Standardösophagektomie bei Patienten mit einem klinischen kompletten Ansprechen („clinical complete response“, cCR) mithilfe einer Clusterrandomisierung prospektiv verglichen. Es werden Patienten randomisiert, die in zwei aufeinanderfolgenden klinischen Beurteilungen des Ansprechens eine cCR zeigen. In der Gruppe der Patienten, die aktiv beobachtet werden, wird im ersten Jahre alle 3 Monate, im zweiten Jahr alle 4 Monate und im dritten Jahr alle 6 Monate eine klinische Kontrolle des Ansprechens vorgenommen. Im Falle eines lokoregionalen Rezidivs wird eine Salvageösophagektomie durchgeführt [21, 22].

Die aktuelle SANO-Studie basiert auf den Daten der vorhergehenden preSANO-Studie. In diese Studie wurden an 6 Zentren 219 Patienten mit Ösophaguskarzinom eingeschlossen, die eine neoadjuvante Radiochemotherapie erhielten. Die Patienten wurden 4 bis 6 Wochen nach Abschluss der neoadjuvanten Therapie klinisch mit Ösophagogastroduodenoskopie (ÖGD) mit Biopsien und Endosonographie untersucht. Bei Patienten ohne residuelle Erkrankung wurde eine zweite Responseuntersuchung mit Positronenemissionstomographie(PET)-Computertomographie(CT), erneuter ÖGD mit Biopsien, Endosonographie und Feinnadelaspiraten verdächtiger Lymphknoten durchgeführt. Bei diesen Patienten wurde 12 bis 14 Wochen nach Abschluss der neoadjuvanten Therapie eine Operation mit Ösophagektomie durchgeführt. $31 \%$ der Patienten mit mehr als $10 \%$ residualem Tumor wurden mit der Endoskopie mit Feinnadelaspiration nicht entdeckt. Mit Bite-on-bite-Biopsien wurden bei $10 \%$ der Patienten mit mehr als $10 \%$ residualem Tumor der Tumor nicht gefunden. In der Endosonographie wurden $28 \%$ und in der PET-CT Untersuchung $15 \%$ nicht gesehen [23]. Mit der preSINO-Studie wird eine analoge Studie aktuell an 4 asiatischen Zentren durchgeführt, um dieses Konzept zu überprüfen [24].

\section{Neue chirurgische Behandlungskonzepte und Technologien}

\section{Chirurgische Konzepte}

Eine entscheidende noch unbeantwortete Frage ist die optimale chirurgische Behandlungsstrategie beim Adenokarzinom des gastroösophagealen Überganges (AEG) II der Kardia. Hierbei wird weiterhin diskutiert, ob Patienten besser mit einer transhiatal erweiterten Gastrektomie oder einer abdominothorakalen Ösophagektomie behandelt werden $[25,26]$. Zur Beantwortung dieser Frage wurde von unserer Klinik der CARDIA Trial aufgelegt und die ersten Patienten dieser multizentrischen internationalen Studie wurden rekrutiert. Ziel dieser Studie ist der Vergleich des Gesamtüberlebens nach transthorakaler IvorLewis-Ösophagektomie und transhiatal erweiterter Gastrektomie [27]. In den letzten Jahren wird zur Behandlung des AEG II im Frühstadium in manchen Zentren zudem die sog. „Doubletrack-reconstruction“-Methode propagiert, welche möglicherweise funktionelle Vorteile bieten könnte [28]. Es liegen aktuell noch keine Ergebnisse aus prospektiv randomisierten Studien, welche dieses Verfahren mit den oben genannten Verfahren für das AEG II bewerten, vor.

\section{Neue chirurgische Technologie}

Hinsichtlich neuer chirurgischer Technologie verweisen wir auf unsere Vorarbeit zu diesem Thema und werden an dieser Stelle nicht im Detail auf die neuen Master-and-Slave-Systeme wie z. B. Medtronic Hugo und roboterähnliche Devices wie z. B. SymphonX eingehen [29]. Insbesondere der immer wieder kritisierte Kostenaspekt wurde in dieser Arbeit auch bereits für unser Zentrum analysiert. Letzteres Device wurde von uns mitentwickelt und die Ergebnisse der Piloteingriffe sind bereits publiziert $[11,30]$.

Die Situation der Roboterchirurgie in Deutschland ist aufgrund mangelnder Refinanzierung der teuren Eingriffe schwierig, diese Situation wird in einer aktuellen Umfrage von Krüger et al. gut zusammengefasst. Insbesondere der obere Gastrointestinaltrakt ist einer der am stärksten wachsende Sektor der roboterassistierten Chirurgie in Deutschland [31].

\section{》) Fluoreszenzlaparoskopie ermöglicht die intraoperative Quantifizierung des Blutflusses}

In Jahre 2021 haben drei Anbieter aus unserer Sicht wesentliche Neuerungen mit möglichen Vorteilen für die chirurgische Therapie des Ösophaguskarzinoms auf den deutschen Markt gebracht. Zum einen das sog. Visionsense-Fluoreszenzlaparoskop der Firma Medtronic als auch das sog. ArtiSential-Instrumentarium der Firma Livsmed und das PinPoint-System der Firma Stryker mit neuem Tool zur Fluoreszenzquantifizierung [12, 32, 33]. Alle genannten Tools sind CE-zertifiziert und kommerziell erwerblich. Das Visionsense-System wird in Köln standardmäBig seit Dezember 2020 im Rahmen der Ivor-Lewis-Ösophagektomie verwendet. Eine Besonderheit ist hier die erstmalig mögliche intraoperative Quantifizierung des Blutflusses mittels Indocyaningrün (ICG). Dies erscheint insbesondere zur Objektivierung oder Vorhersage möglicher Interponatischämien nützlich. Den gleichen Ansatz verfolgt das System von Stryker, hier erfolgt intraoperativ auch eine Kalibrierung des Systems, was möglicherweise einen Zusatznutzen bringt. Zum aktuellen Zeitpunkt kann noch keine kritische Bewertung dieser Systeme erfolgen.

Chirurgisch sehr aussichtsreich insbesondere für Kliniken ohne Zugang zur Robotik erscheint aus unserer Sicht das Instrumentarium von Livsmed, welches roboterähnliche Trian- 

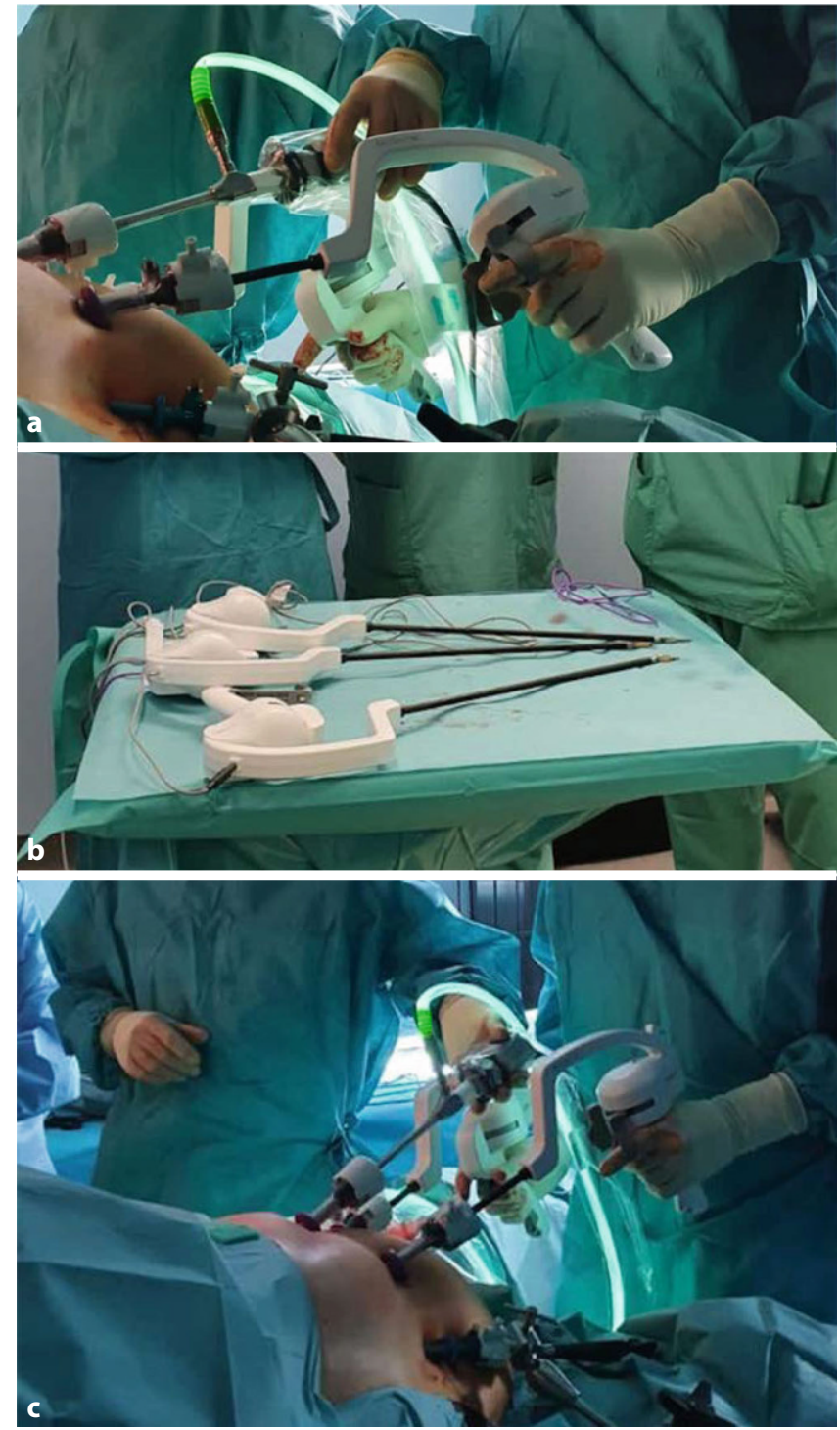

Abb. 1 ArtiSential-Instrumente im Rahmen einer minimal-invasiven IvorLewis-Ösophagektomie in Köln. a Zusammenspiel Fluoreszenzlaparoskopie und Artisential Instrumentarium. b Auswahl an aktuell verfügbarem Instrumentarium. c Beidhändiges Arbeiten mit Artisential erfordert Training

gulation ohne eigentliche Robotiktechnologie ermöglicht. Die Instrumente werden seit Mai 2021 an unserer Klinik verwendet, die Vorteile der Triangulation erscheinen insbesondere bei der Lymphadenektomie in anatomisch schwer zugänglichen Arealen und bei der Rekonstruktion (z. B. Ösophagogastrostomie, Ösophagojejunostomie) evident zu sein. Inwiefern sich hier Kostenersparnisse für bestimmte Eingriffe im Vergleich zu „echten“ Robotern (Master-Slave-Systemen) ergeben können und wie sich die Ergebnisse der Ösophaguschirurgie durch diese Instrumente beeinflussen lassen, muss noch in Studien erarbeitet werden. Sicherlich spielt auch Training bei diesen hochkomplexen laparoskopischen Instrumenten eine Rolle, denn diese lassen sich anders und weniger intuitiv bedienen als beispielsweise aktuell verfügbare Robotersysteme (Abb. 1).

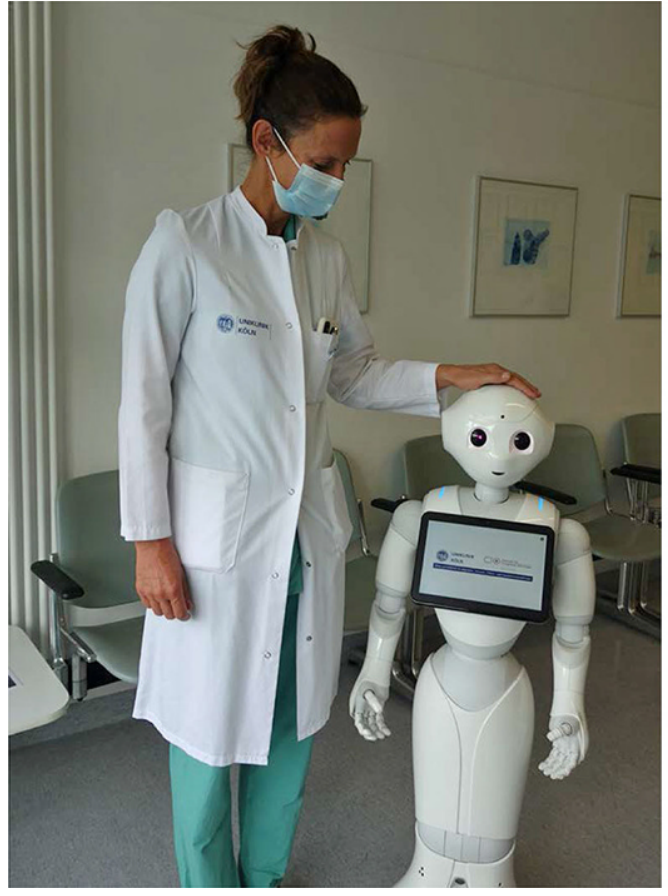

Abb. 2 Humanoider Roboter "Pepper" als Unterstützer bei der Patientenaufklärung im Rahmen der COVID-19-Pandemie. (Foto mit freundlicher Genehmigung C.J. Bruns, Köln)

\section{Humanoide Roboter}

Humanoide Roboter werden aktuell in der Literatur noch ambivalent diskutiert, dennoch steigt deren Einsatz in verschiedenen Gesundheitssystemen [34].

Im Rahmen der COVID-19-Pandemie evaluierten wir an unserem Standort auch den Einsatz humanoider Roboter zur Reduktion von Patientenkontakten im Rahmen der Aufklärung vor Ösophagektomie. Dieses Tool wurde von Patienten und unserem Team gut angenommen, die Ergebnisse einer klinischen Beobachtungsstudie werden in Kürze publiziert (Abb. 2).

\section{Training}

Wie zuvor dargestellt, strömt eine große Menge neuer, meist minimal-invasiver Technologien kombiniert mit neuer Bildgebungstechnik auf den Markt. Diese Technologie soll dem Chirurgen zwar die Arbeit erleichtern und/oder Ergebnisse für den Patienten verbessern, gleichzeitig ist sie aber hochkomplex und erfordert somit Übung. Entscheidend für den Einsatz jedweder neuen chirurgischen Technologie ist optimales Training unter Ausschöpfung virtueller Technologien, aber auch Nutzung von Wetlabs und der Einsatz von Proctoring und Telemedizin. Für die roboterassistierte Ösophagektomie („robot assisted minially invasive esophagectomy“, RAMIE) wurde federführend durch unsere Klinik ein internationaler Delphi-Prozess durchgeführt, welcher nun konkrete Handlungsanweisungen für ein optimales Training der RAMIE vorgibt [10]. Ziel dieses Trainingskurrikulums ist, Lernkurven möglichst schnell zu überwinden, um eine kurze Zeit bis zur Kompetenz („time-to-proficiency“) zu erreichen. In Deutschland wird dieses Trainingskurrikulum über den Berufsverband der Deutschen Chirurgen angeboten [35]. 
Fazit für die Praxis

- Die Therapie des Ösophaguskarzinoms wird weiter zentralisiert und es kommen sowohl innovative neue onkologische als auch primär chirurgische Behandlungskonzepte zum Einsatz.

- Die immer komplexeren Instrumente und Devices erfordern profundes Training, welches in der roboterassistierten Ösophaguschirurgie weiter strukturiert wurde.

- Inwiefern für den Patienten durch die neuen Therapiekonzepte ein Vorteil hinsichtlich der Krankenhausmorbidität oder des Langzeitüberlebens entsteht, muss weiter evaluiert werden.

Literatur

1. Hölscher AH, Gockel I, Porschen R (2019) Aktualisierte S3-Leitlinie zum Ösophaguskarzinom und Ergänzungen aus chirurgischer Sicht [Updated German S3 guidelines on esophageal cancer and supplements from a surgical perspective. Chirurg 90(5):398-402. https://doi.org/10.1007/s00104-019-0954-9

2. Saeki H, Sohda M, Sakai M, Sano A, Shirabe K (2020) Role of surgery in multidisciplinary treatment strategies for locally advanced esophageal squamous cell carcinoma. Ann Gastroenterol Surg 4(5):490-497. https://doi.org/10.1002/ ags3. 12364

3. Eyck BM, van der Wilk BJ, Noordman BJ, Wijnhoven BPL, Lagarde SM, Hartgrink HH, Coene PPLO, Dekker JWT, Doukas M, van der Gaast A, Heisterkamp J, Kouwenhoven EA, Nieuwenhuijzen GAP, Pierie JEN, Rosman C, van Sandick JW, van der Sangen MJC, Sosef MN, van der Zaag ES, Spaander MCW, Valkema $R$ Lingsma HF, Steyerberg EW, van Lanschot JJB, SANO-study group (2021) Updated protocol of the SANO trial: a stepped-wedge cluster randomised trial comparing surgery with active surveillance after neoadjuvant chemoradiotherapy for oesophageal cancer. Trials 22(1):345. https://doi.org/10.1186/s13063-021-05274W

4. Müller-Stich BP, Probst P, Nienhüser H, Fazeli S, Senft J, Kalkum E, HegerP, Warschkow R, Nickel F, Billeter AT, Grimminger PP, Gutschow C, Dabakuyo-Yonli TS, Piessen G, Paireder M, Schoppmann SF, van der Peet DL, Cuesta MA, van der Sluis P, van Hillegersberg R, Hölscher AH, Diener MK, Schmidt T (2021) Meta-analysis of randomized controlled trials and individual patient data comparing minimally invasive with open oesophagectomy for cancer. Br J Surg. https://doi.org/10.1093/ bis/znab278

5. https://www.g-ba.de/downloads/62-492-2546/Mm-R_2021-06-17_iK-202107-29.pdf. Zugegriffen: 17. Aug. 2021

6. Kuppusamy MK, Low DE, International Esodata Study Group (IESG) (2020) Evaluation of international contemporary operative outcomes and management trends associated with esophagectomy: a 4-year study of $>6000$ patients using ECCG definitions and the Online Esodata database. Ann Surg. https://doi.org/10. 1097/SLA.0000000000004309

7. Fuchs HF, Harnsberger CR, Broderick RC, Chang DC, Sandler BJ, Jacobsen GR, Bouvet M, Horgan S (2017) Mortality after esophagectomy is heavily impacted by center volume: retrospective analysis of the Nationwide Inpatient Sample. Surg Endosc 31(6):2491-2497. https://doi.org/10.1007/s00464-016-5251-9

8. Martinello N, Loshak H (2020) Experiences with and expectations of robotic surgical systems: a rapid qualitative review. Canadian Agency for Drugs and Technologies in Health, Ottawa (Internet)

9. Horgan S, Berger RA, Elli EF, Espat NJ (2003) Robotic-assisted minimally invasive transhiatal esophagectomy. Am Surg 69(7):624-626

10. Szold A, Bergamaschi R, Broeders I, Dankelman J, Forgione A, Langø T, Melzer A, Mintz Y, Morales-Conde S, Rhodes M, Satava R, Tang CN, Vilallonga R, European Association of Endoscopic Surgeons. (2015) European Association of Endoscopic Surgeons (EAES) consensus statement on the use of robotics in general surgery. Surg Endosc 29(2):253-288. https://doi.org/10.1007/s00464-014-3916-9

11. Fuchs HF, Collins JW, Babic B, Du Coin C, Meireles OR, Grimminger PP, Read M, Abbas A, Sallum R, Müller-Stich BP, Perez D, Biebl M, Egberts JH, van Hillegersberg $R$,
Bruns CJ (2021) Robotic-assisted minimally invasive esophagectomy (RAMIE) for esophageal cancer training curriculum — a worldwide Delphi consensus study. Dis Esophagus. https://doi.org/10.1093/dote/doab055

12. Datta RR, Dieplinger G, Wahba R, Kleinert R, Thomas M, Gebauer F, Schiffmann L, Stippel DL, Bruns CJ, Fuchs HF (2020) True single-port cholecystectomy with ICG cholangiography through a single 15- $\mathrm{mm}$ trocar using the new surgical platform "symphonX": first human case study with a commercially available device. Surg Endosc 34(6):2722-2729. https://doi.org/10.1007/s00464-019-07229-4

13. Darwich I, Abuassi M, Weiss C, Stephan D, Willeke F (2021) The Artisentia/ ${ }^{\circledR}$ articulated Laparoscopic forceps: a dry lab study to examine dexterity and learning effects in operators with different levels of Laparoscopic experience. Surg Technol Int. https://doi.org/10.52198/21.STI.38.SO1424

14. https://Www.sages.org/meetings/annual-meeting/abstracts-archive/handx-ahand-held-smart-motorized-laparoscopic-device-as-an-alternative-to-roboticsuturing-in-laparoscopic-hernia-repair-first-human-cases/.Zugegriffen: 17. Aug 2021

15. Cancer Genome Atlas Research Network; Analysis Working Group: Asan University; BC Cancer Agency; Brigham and Women's Hospital; Broad Institute; Brown University; Case Western Reserve University; Dana-Farber Cancer Institute; Duke University; Greater Poland Cancer Centre; Harvard Medical School; Institute for Systems Biology; KU Leuven; Mayo Clinic; Memorial Sloan Kettering Cancer Center; National Cancer Institute; Nationwide Children's Hospital; Stanford University; University of Alabama; University of Michigan; University of North Carolina; University of Pittsburgh; University of Rochester; University of Southern California; University of Texas MD Anderson Cancer Center; University of Washington; Van Andel Research Institute; Vanderbilt University; Washington University; Genome Sequencing Center: Broad Institute; Washington University in St. Louis; Genome Characterization Centers: BC Cancer Agency; Broad Institute; Harvard Medical School; Sidney Kimmel Comprehensive Cancer Center at Johns Hopkins University; University of North Carolina; University of Southern California Epigenome Center; University of Texas MD Anderson Cancer Center; Van Andel Research Institute; Genome Data Analysis Centers: Broad Institute; Brown University:; Harvard Medical School; Institute for Systems Biology; Memorial Sloan Kettering Cancer Center; University of California Santa Cruz; University of Texas MD Anderson Cancer Center; Biospecimen Core Resource: International Genomics Consortium; Research Institute at Nationwide Children's Hospital; Tissue Source Sites: Analytic Biologic Services; Asan Medical Center; Asterand Bioscience; Barretos Cancer Hospital; BioreclamationIVT; Botkin Municipal Clinic; Chonnam National University Medical School; Christiana Care Health System; Cureline; Duke University; Emory University; Erasmus University; Indiana University School of Medicine; Institute of Oncology of Moldova; International Genomics Consortium; Invidumed; Israelitisches Krankenhaus Hamburg; Keimyung University School of Medicine; Memorial Sloan Kettering Cancer Center; National Cancer Center Goyang; Ontario Tumour Bank; Peter MacCallum Cancer Centre; Pusan National University Medical School; Ribeirão Preto Medical School; St. Joseph's Hospital \&Medical Center; St. Petersburg Academic University; Tayside Tissue Bank; University of Dundee; University of Kansas Medical Center; University of Michigan; University of North Carolina at Chapel Hill; University of Pittsburgh School of Medicine; University of Texas MD Anderson Cancer Center; Disease Working Group: Duke University; Memorial Sloan Kettering Cancer Center; National Cancer Institute; University of Texas MD Anderson Cancer Center; Yonsei University College of Medicine; Data Coordination Center: CSRA Inc.; Project Team: National Institutes of Health. Integrated genomic characterization of oesophageal carcinoma. Nature. 2017 Jan 12;541(7636):169-175. https://doi.org/ 10.1038/nature20805. Epub 2017 Jan 4. PMID: 28052061; PMCID: PMC5651175. 16. van Hagen $P$, Hulshof MC, van Lanschot JJ, Steyerberg EW, van Berge Henegouwen MI, Wijnhoven BP, Richel DJ, Nieuwenhuijzen GA, Hospers GA, Bonenkamp JJ, Cuesta MA, Blaisse RJ, Busch OR, ten Kate FJ, Creemers GJ, Punt CJ, Plukker JT, Verheul HM, Spillenaar BEJ, van Dekken H, van der Sangen MJ, Rozema T, Biermann K, Beukema JC, Piet AH, van Rij CM, Reinders JG, Tilanus HW, van der Gaast A, CROSS Group (2012) Preoperative chemoradiotherapy for esophageal or 
junctional cancer. N Engl J Med 366(22):2074-2084. https:// doi.org/10.1056/ NEJMoa1112088

17. Al-Batran SE, Homann N, Pauligk C, Goetze TO, Meiler J, Kasper S, Kopp HG, Mayer F, Haag GM, Luley K, Lindig U, Schmiegel W, Pohl M, Stoehlmacher J, Folprecht G, Probst S, Prasnikar N, Fischbach W, Mahlberg R, Trojan J, Koenigsmann M, Martens UM, Thuss-Patience P, Egger M, BlockA, Heinemann V, Illerhaus G, Moehler M, Schenk M, Kullmann F, Behringer DM, Heike M, Pink D, Teschendorf C, Löhr C, Bernhard H, Schuch G, Rethwisch V, von Weikersthal LF, Hartmann JT, Kneba M, Daum S, Schulmann K, Weniger J, Belle S, Gaiser T, Oduncu FS, Güntner M, Hozaeel W, Reichart A, Jäger E, Kraus T, Mönig S, Bechstein WO, Schuler M, Schmalenberg H, Hofheinz RD, FLOT4-AlO Investigators (2019) Perioperative chemotherapy with fluorouracil plus leucovorin, oxaliplatin, and docetaxel versus fluorouracil or capecitabine plus cisplatin and epirubicin for locally advanced, resectable gastric or gastro-oesophageal junction adenocarcinoma (FLOT4): a randomised, phase 2/3 trial. Lancet 393(10184):1948-1957. https://doi.org/10. 1016/S0140-6736(18)32557-1

18. Hoeppner J, Lordick F, Brunner T, Glatz T, Bronsert P, Röthling N, Schmoor C, Lorenz D, EIIC, Hopt UT, Siewert JR (2016) ESOPEC: prospective randomized controlled multicenter phase III trial comparing perioperative chemotherapy (FLOT protocol) to neoadjuvant chemoradiation (CROSS protocol) in patients with adenocarcinoma of the esophagus (NCT02509286). BMC Cancer 16:503. https://doi.org/10.1186/ s12885-016-2564-y

19. Journal of Clinical Oncology 2021, 39, 4004-4004, https://doi.org/10.1200/JCO. 2021.39.15_suppl.4004

20. Kelly RJ, Ajani JA, Kuzdzal J, Zander T, Van Cutsem E, Piessen G, Mendez G, Feliciano J, Motoyama S, Lièvre A, Uronis H, Elimova E, Grootscholten C, Geboes K, Zafar S, Snow S, Ko AH, Feeney K, Schenker M, Kocon P, Zhang J, Zhu L, Lei M, Singh P, Kondo K, Cleary JM, Moehler M, CheckMate 577 Investigators (2021) Adjuvant nivolumab in resected esophageal or Gastroesophageal junction cancer. NEngl J Med 384(13):1191-1203. https://doi.org/10.1056/NEJMoa2032125

21. Noordman BJ, Wijnhoven BPL, Lagarde SM, Boonstra JJ, Coene PPLO, Dekker JWT,

Doukas M, van der Gaast A, Heisterkamp J, Kouwenhoven EA, Nieuwenhuijzen GAP, Pierie JEN, Rosman C, van Sandick JW, van der Sangen MJC, Sosef MN, Spaander MCW, Valkema R, van der Zaag ES, Steyerberg EW, van Lanschot JJB, SANO-study group (2018) Neoadjuvant chemoradiotherapy plus surgery versus active surveillance for oesophageal cancer: a stepped-wedge cluster randomised trial. BMC Cancer 18(1):142. https://doi.org/10.1186/s12885-018-4034-1

22. Eyck BM, van der Wilk BJ, Noordman BJ, Wijnhoven BPL, Lagarde SM, Hartgrink HH, Coene PPLO, Dekker JWT, Doukas M, van der Gaast A, Heisterkamp J, Kouwenhoven EA, Nieuwenhuijzen GAP, Pierie JEN, Rosman C, van Sandick JW, van der Sangen MJC, Sosef MN, van der Zaag ES, Spaander MCW, Valkema R, Lingsma HF, Steyerberg EW, van Lanschot JJB, SANO-study group (2021) Updated protocol of the SANO trial: a stepped-wedge cluster randomised trial comparing surgery with active surveillance after neoadjuvant chemoradiotherapy for oesophageal cancer. Trials 22(1):345. https://doi.org/10.1186/s13063-021-05274w

23. Noordman BJ, Spaander MCW, Valkema R, Wijnhoven BPL, van Berge Henegouwen MI, Shapiro J, Biermann K, van der Gaast A, van Hillegersberg R, Hulshof MCCM, Krishnadath KK, Lagarde SM, Nieuwenhuijzen GAP, Oostenbrug LE, Siersema PD, Schoon EJ, Sosef MN, Steyerberg EW, van Lanschot JJB, SANO study group (2018) Detection of residual disease after neoadjuvant chemoradiotherapy for oesophageal cancer (preSANO): a prospective multicentre, diagnostic cohort study. Lancet Oncol 19(7):965-974. https://doi.org/10.1016/S14702045(18)30201-8

24. Zhang X, Eyck BM, Yang Y, Liu J, Chao YK, Hou MM, Hung TM, Pang Q, Yu ZT, Jiang H, Law S, Wong I, Lam KO, van der Wilk BJ, van der Gaast A, Spaander MCW, Valkema R, Lagarde SM, Wijnhoven BPL, van Lanschot JJB, Li Z (2020) Accuracy of detecting residual disease after neoadjuvant chemoradiotherapy for esophageal squamous cell carcinoma (preSINO trial): a prospective multicenter diagnostic cohort study. Bmc Cancer 20(1):194. https://doi.org/10.1186/s12885-020-6669-y
25. BlankS, Schmidt T, Heger P, Strowitzki MJ, Sisic L, Heger U, Nienhueser H, Haag GM, Bruckner T, Mihaljevic AL, Ott K, Büchler MW, Ulrich A (2018) Surgical strategies in true adenocarcinoma of the esophagogastric junction (AEG II): thoracoabdominal or abdominal approach? Gastric Cancer 21(2):303-314. https://doi.org/10.1007/ s10120-017-0746-1

26. Heger P, Blank S, Gooßen K, Nienhüser H, Diener MK, Ulrich A, Mihaljevic AL, Schmidt T (2019) Thoracoabdominal versus transhiatal surgical approaches for adenocarcinoma of the esophagogastric junction - a systematic review and metaanalysis. Langenbecks Arch Surg 404(1):103-113. https://doi.org/10.1007/s00423018-1745-3

27. Leers JM, Knepper L, van der Veen A, Schröder W, Fuchs H, Schiller P, Hellmich M, Zettelmeyer U, Brosens LAA, Quaas A, Ruurda JP, van Hillegersberg R, Bruns CJ (2020) The CARDIA-trial protocol: a multinational, prospective, randomized, clinical trial comparing transthoracic esophagectomy with transhiatal extended gastrectomy in adenocarcinoma of the gastroesophageal junction (GEJ) type ll. BMC Cancer 20(1):781. https://doi.org/10.1186/s12885-020-07152-1

28. Hölscher AH, Law S (2020) Esophagogastric junction adenocarcinomas: individualization of resection with special considerations for Siewert type ll, and Nishi types $E G, E=G$ and GE cancers. Gastric Cancer 23(1):3-9. https://doi.org/10. 1007/s10120-019-01022-x

29. Urbanski A, Babic B, Schröder W, Schiffmann L, Müller DT, Bruns CJ, Fuchs HF (2021) Neue Techniken und Trainingsmethoden für die roboterassistierte Chirurgie und Kosten-Nutzen-Bewertung anhand der Ivor-Lewis-Ösophagektomie [New techniques and training methods for robot-assisted surgery and cost-benefit analysis of /vor Lewis esophagectomy. Chirurg 92(2):97-101. https://doi.org/10. 1007/s00104-020-01317-1

30. Berducci M, Fuchs HF, Omelanczuk P, Broderick RC, Harnsberger CR, Langert J, Nefa J, Jaureguiberry P, Gomez P, Miranda L, Jacobsen GR, Sandler BJ, Horgan S (2016) Phase Il clinical experience and long-term follow-up using the nextgeneration single-incision platform FMX314. Surg Endosc 30(3):953-960. https:// doi.org/10.1007/s00464-015-4319-2

31. Krüger CM, Rückbeil O, Sebestyen U, Schlick T, Kürbis J, Riediger H (2021) DeRAS Ideutsche Situation der robotisch assistierten Chirurgie - eine Online-Survey-Studie [DeRAS I-German situation of robotic-assisted surgery - an online survey. Chirurg. https://doi.org/10.1007/s00104-021-01404-x

32. Kamada T, Yoshida M, Suzuki N, Takeuchi H, Takahashi J, Marukuchi R, Narihiro S, Ohdaira H, Suzuki Y (2020) Introduction of VISIONSENSE ${ }^{\circledR}$ for indocyanine green fluorescence-guided parathyroidectomy: report of a case. Int J Surg Case Rep 75:418-421. https://doi.org/10.1016/j.ijscr.2020.09.139

33. https://www.stryker.com/us/en/endoscopy/systems/1688-aim-platform.html. Zugegriffen: 17. Aug. 2021

34. Nyholm L, Santamäki-Fischer R, Fagerström L (2021) Users' ambivalent sense of security with humanoid robots in healthcare. Inform Health Soc Care 46(2):218-226. https://doi.org/10.1080/17538157.2021.1883027

35. Fuchs H (2020) Curriculum Robotische Chirurgie im oberen Gastrointestinaltrakt für Oberärzte und leitende Klinikchirurgen. Passion Chir 10(12):Artikel 04_02

Korrespondenzadresse

PD Dr. Hans F. Fuchs

Klinik und Poliklinik für Allgemein-, Viszeral-, Tumor- und

Transplantationschirurgie

Uniklinik Köln

Kerpener Straße 62, 50937 Köln, Deutschland

hans.fuchs@uk-koeln.de

\section{Einhaltung ethischer Richtlinien}

Interessenkonflikt. H. Fuchs: Medtronic: Advisory Board, Activ Surgical: Advisory Board, Fortimedix Surgical: Advisory Board, Intuitive Surgical: 
educational grant. B. Babic: Medtronic: Advisory Board. C. Bruns: Medtronic: Advisory Board. T. Schmidt gibt an, dass kein Interessenkonflikt besteht.

Für diesen Beitrag wurden von den Autoren keine Studien an Menschen oder Tieren durchgeführt. Für die aufgeführten Studien gelten die jeweils dort angegebenen ethischen Richtlinien.

\section{Fachnachrichten}

\section{Curt Meyer-Gedächtnispreis 2022}

Die Berliner Krebsgesellschaft e.V. vergibt den Curt MeyerGedächtnispreis verbunden mit einem Preisgeld in Höhe von 10.000 EUR zur Förderung des wissenschaftlichen Nachwuchses im Land Berlin.

Prämiert wird eine wissenschaftliche Persönlichkeit, die sich mit ihrer Forschungstätigkeit auf dem Gebiet der Krebsmedizin aktuell hervorgetan hat. Durch das Herausstellen von Vorbildern treibt die Berliner Krebsgesellschaft die Spitzenwissenschaft im Bereich der Krebsmedizin voran.

\section{Voraussetzungen}

Der/die Bewerber:in darf nicht älter als 40 Jahre und muss im Land Berlin beruflich tätig sein. Bei Inanspruchnahme von Mutterschutz- und Elternzeiten kann eine Verschiebung der Altersgrenze um zwei Jahre pro Kind gewährt werden.

In einem Begutachtungsverfahren wird der/die Preisträger:in auf der Grundlage von einer oder mehreren hochrangig publizierten Arbeiten bestimmt. Hierfür können Publikationen eingereicht werden, die sich mit Forschungsfragen auf dem Gebiet der klinischen, experimentellen oder translationalen Krebsmedizin sowie der Versorgungsforschung befassen, wenn diese an einer Institution des Landes Berlin erarbeitet wurden. Die Artikel müssen aktuell sein. Wurde das Manuskript einem Verlag zur Veröffentlichung vorgelegt, muss das Begutachtungsverfahren abgeschlossen und das Manuskript zur Veröffentlichung angenommen worden sein. Eine Nominierung ist nicht erforderlich.

Die Vergabe des Preises erfolgt an den/die Erstautor:in. Die Koautor:innen müssen schriftlich bestätigen, dass sie mit der Bewerbung des/der Erstautor:in einverstanden sind.

Die Bewerbung ist an Prof. Dr. med. Petra Feyer, Vorstandsvorsitzende der Berliner Krebsgesellschaft e.V., zu richten. Folgende Unterlagen müssen eingereicht werden:

1. Lebenslauf

2. Wissenschaftlicher Tätigkeitsbericht

3. Publikationsliste

4. Publikation/en, die für die Bewerbung relevant sind

Bewerber:innen senden bitte ihre Unterlagen bis spätestens 31. März 2022 (23.59 Uhr) ausschließlich per E-Mail in einer pdf-Datei (nicht größer als 7MB) an: forschung@berliner-krebsgesellschaft.de

\section{Über den Curt Meyer-Gedächtnispreis}

Der Curt Meyer-Gedächtnispreis gehört zu den renommiertesten Auszeichnungen für junge Krebsforscher:innen in Berlin. 1988 zum ersten Mal von der Berliner Krebsgesellschaft e.V. verliehen, soll der mit 10.000 Euro dotierte Forschungspreis die Spitzenforschung in Berlin stärken und Nachwuchswissenschaftler zu herausragenden Leistungen animieren. Der Preis dient dem ehrenden Gedächtnis an den Berliner Senatsrat Dr. med. Curt Meyer (1891-1984), dessen Name eng mit der Krebsfürsorge verbunden ist. Meyer war Gründungsmitglied und langjähriger Geschäftsführer des Landesausschusses Berlin für Krebsbekämpfung e.V., aus dem 1957 die Berliner Krebsgesellschaft hervorgegangen ist.

Quelle: Berliner Krebsgesellschaft e.V., www.berlinerkrebsgesellschaft.de 
Hier steht eine Anzeige.

Springer 